\title{
Politikberatung im Kontext von Wahlen
}

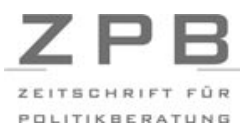

Wahlen sind ein unverzichtbarer Bestandteil eines jeden demokratischen Systems. Auch schlanke Definitionen von Demokratie sehen in Partizipation und Wettbewerb unverzichtbare Elemente, die im politischen System durch Wahlen gewährleistet werden (so etwa Anthony Downs). Es überrascht daher nicht, dass Wahlen auch für die Politikberatung von großem Interesse sind. „Policy advice“ und ,political consulting“ begleiten den gesamten Prozess von Themenfindung und Agenda-Setting über die Kampagnenarbeit bis hin zur Auswertung der Wahlergebnisse und liefern dabei wichtige wissenschaftliche und politisch-praktische Erkenntnisse.

Die vorliegende Ausgabe der ZPB möchte dieses breite Spektrum der „Politikberatung im Kontext von Wahlen“ abbilden und verschiedene Facetten des Themas betrachten. Naturgemäß sind einem solchen Vorhaben mit Blick auf den Umfang des Heftes einerseits und auf die Vielzahl nationaler und internationaler Wahlen andererseits Grenzen gesetzt. Die Beiträge nehmen gleichwohl Wahlkämpfe und Wahlergebnisse, Kommunikationsinstrumente und Strategien, Forschungsergebnisse und Anwendungshinweise in den Blick und können somit einen Eindruck von der Vielfalt der Beratungslandschaft im Kontext von Wahlen vermitteln.

Gleich der erste Aufsatz widmet sich einem Thema, das ebenso präsent wie unerforscht ist: Wahlplakate. Stephanie Geise beschreibt die Plakatkampagnen der fünf großen Parteien zur Bundestagswahl 2009 und stellt fest, dass die Potenziale dieser Kommunikationsform nur in Teilen genutzt wurden. Im Anschluss daran befasst sich Niko Switek mit den jüngsten Wahlausgängen auf Bundes- und insbesondere auch Landesebene, die ein ums andere Mal die Notwendigkeit neuer Koalitionsmodelle zeigen. Unter Einbeziehung der strategischen Dimension von Koalitionsbildungsprozessen lotet er die Chancen für vier neue Koalitionsmodelle auf Bundesebene aus. Uwe Jun und Johannes Pütz berichten aus dem Innenleben der SPD-Kampagne und untersuchen die strategischen Planungsprozesse im Vorfeld des TV-Duells 2009 zwischen Angela Merkel und Frank-Walter Steinmeier. Dabei verdeutlichen sie die Notwendigkeit einer klaren Struktur und zeigen Schwachstellen der internen Wahlkampfberatung auf.

Die Analyse und Verarbeitung von Wahldaten ist Thema der beiden Beratungsporträts. Christina Eder, Manuela S. Kulick und Christof Wolf stellen die Arbeit des Forschungsdatenzentrums „Wahlen“ am GESIS - Leibniz-Institut für Sozialwissenschaften vor, die sich der wissenschaftlichen Aufbereitung von Wahl- und Umfragedaten widmet. Das SORA Institute for Social Research and Consulting richtet seine Wahl- und Politikforschung ebenso wie die daraus hervorgehenden Beratungsleistungen an Kunden aus

Online publiziert: 21.10 .2010

(C) VS Verlag für Sozialwissenschaften 2010 
Interessensvertretungen, öffentlichen Einrichtungen und Privatunternehmen, wie Florian Oberhuber und Christoph Hofinger darstellen. Im Anschluss widmen sich die beiden Praxisberichte den personenbezogenen Komponenten des Wahlkampfes. Jan Blume, Lars Harden und Annika Heisig zeigen mit Hilfe von Medienimages, wie Amtsinhaber Horst Köhler und Herausfordererin Gesine Schwan im Vorfeld zur Wahl des Bundespräsidenten wahrgenommen wurden. Und Olaf Bock und Roland Willner schildern Erfahrungen aus einem universitären Beratungsprojekt zur SPD-Erststimmenkampagne in HamburgAltona. Die Beiträge im Forum erweitern die Perspektive in zweierlei Hinsicht: Maik Bohne analysiert die zahlenmäßig große und doch meist nur am Rande erwähnte Gruppe der Nichtwähler in Deutschland und Henrik Flor zieht ein Jahr nach dem erfolgreichen und vielfach als wegweisend beschriebenen Wahlkampf von Barack Obama ein erstes Fazit zu der Frage, wie neue Medien Wahlkämpfe und Entscheidungsprozesse in den USA verändern. Im Literaturteil bespricht Susumu Shikano den Ansatz einer neuen politischen Psychologie im Sammelband von Thorsten Faas, Kai Arzheimer und Sigrid Roßteutscher „Information - Wahrnehmung - Emotion“. Der Wahlkampf Barack Obamas ist Thema der Rezension von Andreas Hoffelder zur Herausgeberschaft der Bertelsmann Stiftung „Obama und das Internet - der neue Weg zum Wahlerfolg?“. Zudem erscheint in der vorliegenden Ausgabe zum ersten Mal ein internationaler Pressespiegel, zusammengestellt von Abhinav Verma. Den Schluss bilden wie gewohnt die Corners, unter anderem mit einem Bericht zu den Debatten um den Nichtraucherschutz.

Wir wünschen allen Leserinnen und Lesern eine anregende Lektüre und interessante Erkenntnisse beim Erkunden der „Politikberatung im Kontext von Wahlen“.

Herausgeber und Redaktion 